\title{
A Review of Ubiquitous Mobile Sensing Based on Smartphones
}

\author{
Chih-Yen Chen ${ }^{1}$, Yung-Hsiang Chen ${ }^{1}$, Chun-Fu Lin ${ }^{1,2}$, \\ Chun-Jen Weng1,3, and Hung-Chun Chien ${ }^{4, *}$ \\ ${ }^{1}$ Instrument Technology Research Center, National Applied Research Laboratories, Taiwan \\ ${ }^{2}$ Institute of Electrical Control Engineering, National Chiao Tung University, Taiwan \\ ${ }^{3}$ Department of Photonics \& Institute of Electro-Optical Engineering, National Chiao Tung University, Taiwan \\ ${ }^{4}$ Department of Electronic Engineering, Jinwen University of Science and Technology, Taiwan \\ (Received 23 September 2013; Accepted 9 January 2014; Published on line 1 March 2014) \\ *Corresponding author: hcchien@just.edu.tw \\ DOI: $10.5875 /$ ausmt.v4i1.301
}

\begin{abstract}
Smartphones or mobile phones are rapidly becoming a popular device for communication, sensing and access of the network system in people's lives. The most significance is that today's smartphones provide a programmable computing platform with the built-in sensors to create many useful applications in the personal group and community. We believe whether built-in sensors or the newly external sensors equipped with smartphones will change many parts of our industrial manufacturing, environmental monitoring, biology and medicine application, healthcare and social network services. In this review paper, we surveyed the existing sensing devices for various applications to improve the functionality. So we explored the emerging sensing paradigms, and several newly developed innovations to formulate an appropriate framework for mobile devices. Finally, the future challenges arising in the mobile sensing and the implementation of instrumentation and measurement in actual situations were discussed.
\end{abstract}

Keywords: Mobile; Smartphone; Sensor; Monitor; Measurement

\section{Introduction}

Since the mobile phone was created and made by a Motorola in 1973, it has really changed our lifestyles drastically [1]. Specifically, a smartphone or a mobile phone is built on a mobile operating system to offer advanced computing capability and connectivity.

Because more and more smartphones are attracting people by the continuous extension of functions and the reduction of their size, such products are becoming one of the fastest growth products in the technology industry. The primary role of smartphones is not only to transmit our voice to other people in anywhere and at anytime, but to catalyze convenient services and peripheral products [2]. According to the recent report of International Data Corporation (IDC), the worldwide smartphones are forecast to grow $7.3 \%$ year over year in 2013, outperforming the broader index from the nearly flat (1.2\%) growth experienced in 2012. A robust growth in the worldwide smartphones shipments are expected to contribute almost 1 billion units for the first time in a single year because of the increasing market demand for smartphones [3].

With the rapid advances in smartphone technology, the operating system is permiting efficient computation for users. Besides, the other advents of the smartphones are driven by equipping one or more powerful sensors, like microphone, camera, an accelerometer, gyroscope, compass, GPS, and ambient light sensor $[2,4,5]$, which can be operated by the application softwares, to satisfy the users demand and create the customer value. Further, the accessibility of ubiquitous wireless network extends the application oriented services with existing sensing capabilities in the portable and feasible platform. So that, more and more people are trying to analyze the sensing data corresponding to regions for the 
applications full of evolutions, opportunities and challenges.

Most of the previous studies have focused on newly developed built-in sensors, but it has also been demonstrated that external sensors equipped with the smartphones is a potentially growing trend [5-7]. With such developments, it may help facilitate the customization and meet the specific needs, e.g. environmental monitoring, healthcare, remote control and social network and community at all times and places. Many reasons indicated the smartphones would be an excellent solution for sensing application in the future. The first reason is that the systems provide a standardized architecture that promotes rapid, low cost, compatibility and the basic operating system, and this will reduce the investment of infrastructure. Second, the popularity of smartphones and wireless network with the benefits of a ubiquitous mobile computing device, and various sensors in everywhere, make the sensing processes easy to be implemented. Third, portability, miniaturisation made the smartphones for low-power consumption and prolonged use as the best platform of sensor carriers.

Chih-Yen Chen received the MS degree from the Department of Physics, at National Taiwan Normal University and Ph.D. from the Institute of Biomedical Engineering, National Yang-Ming University in 2003 and 2010, respectively. Now, he is an assistant researcher at the Instrument Technology Research Center, National Applied Research Laboratories, from 2011 to present. His research interests include image processing, computer-aided diagnosis and human machine intelligence.

Yung-Hsiang Chen received his M.S. from the Department of Electrical Engineering, National Changhua University of Education. He is currently an associate engineer at Instrument Technology Research Center (ITRC), National Applied Research Laboratories (NARL), Taiwan. His research areas of interest include the image processing analysis, machine vision, and opto-mechanical systems design, etc.

Chun-Fu Lin received the B.S. and M.S. degree in electronic engineering from Yuan Ze University and Chung Yuan Christian University in 2001 and 2003, respectively. Since 2010 he has been an associate researcher in Instrument Technology Research Center, National Applied Research Laboratories, Taiwan. He is currently a Ph.D. student in the Institute of Electrical Control Engineering at National Chiao Tung University. His current research interests include circuit theory, image processing, image recognition, intelligent system design and computer vision.

Chun-Jen Weng has worked at the Instrument Technology Research Center (ITRC) of National Applied Research Laboratories (NARL) for 12 years. He is an associate researcher. He has been conducting research on broadband spectroscopy application and micro-optics design and measurement.

Hung-Chun Chien was born in New Taipei City, Taiwan, in 1978. He received the PhD degree in Electronic Engineering from National Taiwan University of Science and Technology (NTUST), Taipei, Taiwan, in 2007. In 2003, he joined Chung-Shan Institute of Science and Technology (Materials and Electro-Optics Research Division), Lung-Tan, Taiwan, as an Assistant Researcher, where he worked in the area of analog circuit and laser technologies. From 2007-2008, he served as a post-doctoral researcher in the Department of Electronic Engineering of National Taiwan University of Science and Technology. Since 2008, he was with the Department of Electronic Engineering, Jinwen University of Science and Technology, New Taipei City, Taiwan, as an Assistant Professor. His teaching and research interests are in the areas of current-mode circuits, analog oscillators and filters, and analog signal processing applications. Dr. Chien is an Editorial Board Member of ISRN Electronics.
This paper explores the sensing applications for the smartphones with built-in sensors or external sensors and communication capabilities for collecting sensor readings, which serve as computing benchmarks for the implementation of advanced measurement and processing information using smartphones. We also discussed a number of leading application areas and sensing paradigms that have emerged in the literatures recently.

The rest of the paper is organized in the following way. The second section describes the built-in sensors and some external sensors. The third section presents the equipped sensors on their application fields. The fourth section gives conclusion and directions for the future marks.

\section{Sensors and potential applications}

Nowadays, smartphones are acting as the sensor carriers and interpreting information connected from machines, instrument or external sensors. Due to the recent development of sensing devices, they are used to extend the new functions for flexibility. More importantly, higher accessible network system and increased bandwidth enables more data to be shared at any time. The support of open and programmable platform of smartphones improves both practicability and convenience for developers to study new sensing applications [5]. The subsequent discussion will introduce the built-in sensors and external sensors, and explain how users control their devices and display content.

\section{Built-in Sensors}

In general, the smartphones are composed of a screen, a microphone, cameras, an accelerometer, GPS receiver, a digital compass, a gyroscope and a communication module. In table 1 , these sensors with the original function, technology and possible applications are listed item by item $[5,6]$. For a microphone, it can make a use of voice input replacing text message or commands for operation, a camera is used to snap pictures and videos, a tri-axis accelerometer can detect motion on three different axes to change the display mode or interpret sudden motions, the gyroscope and digital compass are integrated to perform specific tasks of navigation when GPS signals are weak or not present, and an ambient light sensor is typically used for adjusting the brightness of the screen backlight hence to a comfortable screen brightness and saving battery energy. Among the items quoted in Table 1 , nine common built-in sensors in the smartphones are presented. 
Table 1. Functions of built-in sensors and the relevant applications.

\begin{tabular}{|c|c|c|c|}
\hline Sensor & Function & Technology & Application \\
\hline Accelero-meter & Rotating Screen & Acceleration & Vibration Sensors \\
\hline Ambient light & Light sensor & Detecting of light intensity & $\begin{array}{c}\text { Screen brightness adjustment \& } \\
\text { energing }\end{array}$ \\
\hline Camera & Taking picture \& video & Recording of scene & Image analysis \& colorimetry \\
\hline Digital compass & Compass & Measuring magnetic fields by & hall effect \\
\hline Gyroscope & Postures of mobile device & $\begin{array}{c}\text { Measuring angular rate by } \\
\text { Coriolis effect }\end{array}$ & Determination for position \& \\
\hline GPS & GPS receiver & Radio Frequency & Position \& Clock \\
\hline Microphone & Conducting voice & Sound & Spectrum analyzer for sound \& \\
\hline NFC & RFID & Near field communication & Payment, ticketing \& security \\
\hline Proximity & Photoelectric sensor & Detecting distance & turn off screen \\
\hline
\end{tabular}

Table 2. Functions of external sensors and the relevant applications.

\begin{tabular}{|c|c|c|}
\hline Sensor & Function & Application \\
\hline Chemical sensors & Detection of chemical \& gas ingredients & Breathalyzer, perfume matching, food freshness \\
\hline Physiological sensors & Detection of physiological signal & Healthcare, diagnosis, body examination \\
\hline 3D cameras & Depth decision & Augmented reality, gesture control \\
\hline Microbolometer & Infrared spectrum & Infrared image \& heat detection \\
\hline Barometer & Altitude & Targeting \\
\hline Magnetometers & Orientation & Directional navigation \\
\hline
\end{tabular}

\section{External Sensors}

For now, smartphones are easily available programmable devices with various functionalities by operating the application softwares to control the built-in sensors. Despite the prominent properties of smartphones, there still existed a lot of problems to restrict the sensing of environment, physiology and healthcare. Fortunately, some external sensors are developed to provide the detection tasks, such as air pollution, temperature, humidity, air pressure, chemicals, physiology signal, and cardiovascular. The concept is not only extending the usability of smartphones but the sensing capabilityanywhere and anytime. Based on the requirement, the approaches of how smartphones can be equipped with more features as an instrumentation tool for measurement and monitoring will be introduced in details. Some external sensors are listed in Table 2 for the explanations [8].

\section{Examples of State of the art}

The integration of the sensing devices, mobile computation and wireless communication, will embrace new products and services with the convenience. In the present more than before, the new technology promotes the great advances in mobile computing and truly impact on our daily lives. Further, the applications consist of participatory sensing processes which require active user's involvement to share the information as the big data $[5,9]$. The following is the overview of the main application areas and sensing paradigms that have emerged in the recent developments.

\section{Applications for environment monitoring}

The static sensing infrastructures, such as environmental sensors, positioning sensors, and energy consumption, are becoming more prevalent so that the 
monitoring and detecting real-world events has become feasible and available for everyone $[10,11]$.

Environmental sensors can be placed both in outdoor and indoor sites for showing environmental factors. For example, some gas sensors imply to monitor specific compositions of $\mathrm{CO}_{2}, \mathrm{O}_{2}$ and $\mathrm{H}_{2}$ in air [7]. In most situations, this kind of environmental sensors are demanded with the characteristics of low power and low cost. The actual deployments of mobile sensing systems should be considered the adjustable duty cycle while maintaining the necessary application fidelity by the low consumption algorithms for long-term monitoring. The power supply to the sensors and smartphones could be charged with electricity for the indoor facilities and urban district, and with a storage battery for suburban district. In the paper of [12], the study was conducted to measure temperature in airport and hospital by installing temperature sensing mobile robot. The demonstration shows the robot was very helpful for the heat temperature detection for firefighting. Monitoring temperature in vehicles or boiler is another practical use for warning earlier [13].

Microphone is an essential component of phones, and sound measurements can be implemented by analyzing the received data. An assessment of phonemes provides an interesting alternative to pure tones in hearing tests [14]. They propose a self-hearing test for the four Korean phonemes. Another study presents interesting experiments that describing the different sound types, and it enables listeners to discriminant these differences [15]. The use of sensing device can be applied to measure the occupational noise exposure by the microphone and GPS sensor for calculating sound diffusion [16].

A colorimetric method is a well-known basic concept, and it can be attained by incorporating a smartphone camera for color analysis. As the measurement of proton concentration, it was conducted on the color detection of $\mathrm{pH}$ paper for showing the chemical change [17]. Similarly, the evaluation of the amount of chlorine in water is based on the camera sensing to obtain the chlorine concentration [18]. Furthermore, there are some applications for replacing spectroscopic analysis of foods and beverages [19] and for exciting the Rhodamine 123 doped within a Mesostructured silica sphere [20].

\section{Applications of Biology and Medicine}

Rapid advances of smartphones made the computer vision, image processing, and signal processing possible to perform the computation on the local platform. In the application of solving agriculture problems, the judgment of plant disease can be made by calculating the insect number and disease areas in the segmented images of the leaves [21]. Appropriate level of banana ripeness is to analyze two broad-spectral images of the banana image under white light [22].

The smartphones built-in cameras are usually providing limited image quality. Therefore, the handyscope product brings smartphones into medical field, converting it into a digital dermatoscope for mobile skin examinations [4]. In the investigation of skin features, skin images can be captured for analysis with image processing procedures to generate the roughness and wrinkle characteristics [6]. As shown is the micro-vessel with immersion oil applied between the lens and the nail, a smartphone make it possible to act like the microcirculation monitoring instrument [7].

A new innovation of optical technologies, named Optical Farming, is introduced by optical sensing instruments via a sensor network system to transmit the collect data on agricultural products growing in fields. One of the devices is smartphone based spectroscopy. It is easy to be found the existing physiological effect on plant growth [23]. For the clinical use, the focused ultrasonography was developed in quantifying airway for the prediction of difficult intubation [24].

\section{Applications of Healthcare}

From real-time patient monitoring to healthcare in daily life, smartphones play a vital role in fulfilling the practice of healthcare anytime and anywhere. In this review, we introduce the recent studies to show how the smartphone has changed the field appreciably and their development.

Physiology sensing devices include the monitor of heart rate, body temperature, respiration, blood pressure, motion/acceleration, blood glucose, ECG, and etc. The prototype of a wireless blood flow velocity monitoring system that provides a home self-monitoring healthcare device for patients in a long-term of peripheral blood flow dynamics and blood flow velocity $[25,26]$. Another paper proposes a real-time ECG transmission for high risk cardiac patients via smartphones system to direct access in remote area. Once the person needs emergent help, the smartphone can automatically notice preassigned caregivers or call the ambulance [27]. To assess physiological status in the environmental or athlete data, a continuous multi-sensor monitoring system (activities, travel, exercise, and food consumption) was offered to real-time feedback test for training improvement [28].

Most of the sophisticated computation of intensive signal algorithms should be run on a cloud server which enables recognition of the role of daily life on health for preventing high risks, prediction response 
to some diseases, and self-management of patients. Therefore, a data relaying through smartphone is the best way to provide anytime health management. Then, a lot of physiological signals could be transmitted to a cloud server via some wireless data transmission equipped on smartphones, such as Bluetooth, Zigbee, Wifi, 3G, or even the LTE, to become more easier to carry around with one. Another potential application is that smartphones deliver alert and warning messages to medical institutions in emergency situations.

\section{Social Network and Community}

Nowadays, smartphones have become sophisticated communicating and portable sensing devices that can be used for creating powerful mobile sensor network. Except for being sensor carriers, smartphones are also communication nodes in a social network thus enabling the share of the sensor readings. With increasing number of developed application software, the general behaviors or preferences are easily acquired to reveal more interesting experiences. This procedure both collects sensor readings and contributes users' sensations, observations and opinions, i.e. human activity [29]. It is notable that such sensor readings are usually associated with the location. The tracking technologies by GPS are providing discreet spatio-temporal sensor readings that can produce additional information for users. For instance, if smartphones are the temperature and humidity sensors, users can obtain accurate weather information in certain areas from the sensor readings of the local community. That means the gathering data about people and their environments brings a new opportunity for people to discover human social activities.

\section{Conclusion and future remarks}

These article overviews the current state and the following challenges in the emerging field of smartphones sensing applications. The integration of sensors, application softwares, and the smartphones with wireless network has the potential for the measurement and instrumentation tools in anytime and anywhere. With benefits of portability and feasibility, the smartphones will cause dynamic sensing processes more useful to match applications in actual condition of living or working areas for the human being. To lower the power consumption, the recording and uploading data may be used intermittently. Furthermore, since the wireless charging technology is becoming more and more polular, it can provide the uninterruptible energy supply for the sensing applications.
Recently, people are trying to interpret the accumulation of vast and complex information databases which is also referred to as "Big Data" relevant to our habits or the meaningful contents. Many technologies of data processing are developed to sort context, extract the nature of knowledge, and discover the hidden pattern with strong but unexpected correlation. That means different sensing signals from the sensor network can be acquired in a flexible manner to comprehend the level of information content depended on. At the same time, the sensors will creat sensing activities at multiple scales and return opportunistically feedback to the users.

As smartphones have been widely prevalent, the cost is expected to be further reduced, and the perform of environment sensing, biology and medicine application, healthcare monitoring and many other aspects, are much easier to achieve. This fact can also boost motivations of more engineers and researchers worldwide in the sensing topic on smartphones system. The future research on the matter will involve useful and effective feedback to users. We believe smartphones sensing systems will create a big impact on our daily life and help define new functions as a whole.

\section{References}

[1] J. J. Andover, "Mobile radio: Sparks fly," Spectrum, IEEE, vol. 10, no. 9, pp. 69-69, 1973. doi: 10.1109/MSPEC.1973.5212836

[2] A. Klausner, A. Trachtenberg, D. Starobinski, and M. Horenstein, "An overview of the capabilities and limitations of smartphone sensors," International Journal of Handheld Computing Research (IJHCR), vol. 4, no. 2, pp. 69-80, 2013. doi: 10.4018/ihcr.2013040105

[3] K. Restivo, R. Llamas, and M. Shirer, Worldwide mobile phone market forecast to grow $7.3 \%$ in 2013 driven by 1 billion smartphone shipments, according to idc, 2013, [Online].

Available:

http://www.idc.com/getdoc.jsp?containerld=prUS 24302813

[4] D. Hofmann, P. G. Dittrich, E. Duntsch, and D. Kraus, "Smart instrumentation for mobile diagnosis and quality assurance in industry, biology and medicine," Biomed Tech (Berl), 2012.

doi: 10.1515/bmt-2012-4291

[5] N. D. Lane, E. Miluzzo, L. Hong, D. Peebles, T. Choudhury, and A. T. Campbell, "A survey of mobile phone sensing," IEEE Communications Magazine, vol. 48, no. 9, pp. 140-150, 2010. doi: $10.1109 /$ MCOM.2010.5560598 
[6] C. C. Wu, S. J. Chou, T. S. Liao, C. H. Hwang, and D. P. Tsai, "Human facial skin roughness and wrinkle inspection based on a smart phone-a preliminary study," presented at the 5th WACBE World Congress on Bioengineering, Tainan, Taiwan, 2011.

[7] S. Aram, A. Troiano, and E. Pasero, "Environment sensing using smartphone," in IEEE Sensors Applications Symposium (SAS), Brescia, Italy, 2012, pp. 1-4.

doi: 10.1109/SAS.2012.6166275

[8] J. A. Ask, "The future of mobile is user context," Forrester research, 2011.

[9] N. Davidovic, D. Rančić, and L. Stoimenov, "Ardsense: Extending mobile phone sensing capabilities using open source hardware for new citizens as sensors based applications," presented at the 16th AGILE Conference on Geographic Information Science, Leuven, Belgium, 2013.

[10] Z. Daqing, G. Bin, and Y. Zhiwen, "The emergence of social and community intelligence," Computer, vol. 44, no. 7, pp. 21-28, 2011. doi: $10.1109 / M C .2011 .65$

[11] J. Yun, S.-S. Lee, I.-Y. Ahn, M.-H. Song, and M.-W. Ryu, "Monitoring and control of energy consumption using smart sockets and smartphones," in Computer applications for security, control and system engineering. vol. 339, T.-h. Kim, A. Stoica, W.-c. Fang, T. Vasilakos, J. Villalba, K. Arnett, M. Khan, and B.-H. Kang, Eds.: Springer Berlin Heidelberg, 2012, pp. 284-290. doi: $10.1007 / 978-3-642-35264-538$

[12] M. F. L. Abdullah and L. M. Poh, "Mobile robot temperature sensing application via bluetooth," International Journal of Smart Home, vol. 5, no. 3, p. 39, 2011.

[13] Q. Shan and D. Brown, "Wireless temperature sensor using bluetooth," in International Workshop on Wireless Ad-hoc Networks, London, UK, 2005.

[14] C. Jong Min, S. Junil, K. Yunseo, K. Dongwook, and L. Junghak, "Phoneme-based self hearing assessment on a smartphone," IEEE Journal of Biomedical and Health Informatics, vol. 17, no. 3, pp. 526-529, 2013. doi: $10.1109 / \mathrm{JBHI} .2013 .2238549$

[15] J. Kuhn and P. Vogt, "Analyzing acoustic phenomena with a smartphone microphone," The Physics Teacher, vol. 51, no. 2, pp. 118-119, 2013. doi: $10.1119 / 1.4775539$

[16] R. Dumoulin and J. Voix, "Calibration of smartphone-based devices for noise exposure monitoring: Method, implementation, and uncertainties of measurement," The Journal of the Acoustical Society of America, vol. 133, no. 5, pp. 3317-3317, 2013. doi:10.1121/1.4805531
[17] B. Y. Chang, "Smartphone-based chemistry instrumentation: Digitization of colorimetric measurements," Bulletin of the Korean Chemical Society, vol. 33, no. 2, pp. 549-552, 2012. doi: $10.5012 /$ bkcs.2012.33.2.549

[18] S. Sumriddetchkajorn, K. Chaitavon, and Y. Intaravanne, "Mobile device-based self-referencing colorimeter for monitoring chlorine concentration in water," Sensors and Actuators B: Chemical, vol. 182, no. 0, pp. 592-597, 2013.

doi: $10.1016 /$ i.snb.2013.03.080

[19] Z. Iqbal and R. B. Bjorklund, "Assessment of a mobile phone for use as a spectroscopic analytical tool for foods and beverages," International Journal of Food Science \& Technology, vol. 46, no. 11, pp. 2428-2436, 2011. doi: 10.1111/i.1365-2621.2011.02766.x

[20] J. Canning, A. Lau, M. Naqshbandi, I. Petermann, and M. J. Crossley, "Measurement of fluorescence in a rhodamine-123 doped self-assembled "giant" mesostructured silica sphere using a smartphone as optical hardware," Sensors, vol. 11, no. 7, pp. 7055-7062, 2011.

Available:

http://www.mdpi.com/1424-8220/11/7/7055

[21] K. Wang, S. Zhang, Z. Wang, Z. Liu, and F. Yang, "Mobile smart device-based vegetable disease and insect pest recognition method," Intelligent Automation \& Soft Computing, vol. 19, no. 3, pp. 263-273, 2013. doi: $10.1080 / 10798587.2013 .823783$

[22] Y. Intaravanne, S. Sumriddetchkajorn, and J. Nukeaw, "Cell phone-based two-dimensional spectral analysis for banana ripeness estimation," Sensors and Actuators B: Chemical, vol. 168, pp. 390-394, 2012. doi: $10.1016 /$ j.snb.2012.04.042

[23] Y. Saito and K. Kobayashi, "Proposal of optical farming: Development of several optical sensing instruments for agricultural use," in Proceeding SPIE 8881, Sensing Technologies for Biomaterial, Food, and Agriculture, Yokohama, Japan, 2013, vol. 8881, p. 888109. doi: $10.1117 / 12.2028830$

[24] J. Wojtczak and P. Bonadonna, "Pocket mobile smartphone system for the point-of-care submandibular ultrasonography," Am J Emerg Med, vol. 31, no. 3, pp. 573-577, 2013. doi: 10.1016/j.ajem.2012.09.013

[25] J. Merilahti, J. Parkka, K. Antila, P. Paavilainen, E. Mattila, E. J. Malm, A. Saarinen, and I. Korhonen, "Compliance and technical feasibility of long-term health monitoring with wearable and ambient technologies," J Telemed Telecare, vol. 15, no. 6, pp. 302-309, 2009. doi: $10.1258 /$ itt.2009.081106 
[26] W. Chih-Chieh, C. Chien-Hung, L. Tai-Shan, and H. Chi-Hung, "Home self-monitoring of blood flow velocity based on labview mobile environment," in IEEE Instrumentation and Measurement Technology Conference (I2MTC), 2011, pp. 1-3. doi: 10.1109/IMTC.2011.5944095

[27] V. Gay and P. Leijdekkers, "A health monitoring system using smart phones and wearable sensors," International Journal of $A R M$, vol. 8, no. 2, pp. 29-35, 2007.

[28] S. T. Doherty and P. Oh, "A multi-sensor monitoring system of human physiology and daily activities," Telemed J E Health, vol. 18, no. 3, pp. 185-192, 2012.

doi: $10.1089 /$ tmj.2011.0138
[29] V. K. Varadan, P. S. Kumar, P. Rai, N. Banerjee, R. E. Harbaugh, S. Oh, and H. Kwon, "E-nanoflex sensor system: Smartphone-based roaming health monitor," Journal of Nanotechnology in Engineering and Medicine, vol. 2, no. 1, pp. 011016-011016, 2011. doi: $10.1115 / 1.4003479$

[30] M. F. Goodchild, "Citizens as sensors: The world of volunteered geography," GeoJournal, vol. 69, no. 4, pp. 211-221, 2007. doi: $10.1007 / \mathrm{s} 10708-007-9111-\mathrm{y}$ 